\title{
A novel ICK mutation causes ciliary disruption and lethal endocrine-cerebro-osteodysplasia syndrome
}

\author{
Machteld M. Oud ${ }^{1 \dagger}$, Carine Bonnard ${ }^{2 \dagger}$, Dorus A. Mans ${ }^{1 \dagger}$, Umut Altunoglu ${ }^{3}$, Sumanty Tohari ${ }^{4}$, Alvin Yu Jin Ng ${ }^{4}$, \\ Ascia Eskin ${ }^{5}$, Hane Lee ${ }^{6}$, C. Anthony Rupar ${ }^{7,8,9}$, Nathalie P. de Wagenaar ${ }^{1}$, Ka Man Wu$^{1}$, Piya Lahiry ${ }^{10}$, \\ Gregory J. Pazour ${ }^{11}$, Stanley F. Nelson ${ }^{5,6}$, Robert A. Hegele ${ }^{7,12}$, Ronald Roepman ${ }^{1}$, Hülya Kayserili 3,13, \\ Byrappa Venkatesh ${ }^{4}$, Victoria M. Siu ${ }^{7,8,9}$, Bruno Reversade ${ }^{2^{*}+\dagger}$ and Heleen H. Arts ${ }^{1,7,9,12^{*}+\dagger}$
}

\begin{abstract}
Background: Endocrine-cerebro-osteodysplasia (ECO) syndrome [MIM:612651] caused by a recessive mutation (p.R272Q) in Intestinal cell kinase (ICK) shows significant clinical overlap with ciliary disorders. Similarities are strongest between ECO syndrome, the Majewski and Mohr-Majewski short-rib thoracic dysplasia (SRTD) with polydactyly syndromes, and hydrolethalus syndrome. In this study, we present a novel homozygous ICK mutation in a fetus with ECO syndrome and compare the effect of this mutation with the previously reported ICK variant on ciliogenesis and cilium morphology.
\end{abstract}

Results: Through homozygosity mapping and whole-exome sequencing, we identified a second variant (c.358G > T; p.G120C) in ICK in a Turkish fetus presenting with ECO syndrome. In vitro studies of wild-type and mutant mRFP-ICK (p.G120C and p.R272Q) revealed that, in contrast to the wild-type protein that localizes along the ciliary axoneme and/or is present in the ciliary base, mutant proteins rather enrich in the ciliary tip. In addition, immunocytochemistry revealed a decreased number of cilia in ICK p.R272Q-affected cells.

Conclusions: Through identification of a novel ICK mutation, we confirm that disruption of ICK causes ECO syndrome, which clinically overlaps with the spectrum of ciliopathies. Expression of ICK-mutated proteins result in an abnormal ciliary localization compared to wild-type protein. Primary fibroblasts derived from an individual with ECO syndrome display ciliogenesis defects. In aggregate, our findings are consistent with recent reports that show that ICK regulates ciliary biology in vitro and in mice, confirming that ECO syndrome is a severe ciliopathy.

Keywords: Endocrine-cerebro-osteodysplasia syndrome, ECO, Intestinal cell kinase, ICK, Short-rib thoracic dysplasia syndrome, SRTD, Ciliopathy, Ciliary defects

\footnotetext{
*Correspondence: bruno@reversade.com; heleen.arts@radboudumc.nl;

harts@robarts.ca

${ }^{\dagger}$ Machteld M. Oud, Carine Bonnard, and Dorus A. Mans contributed

equally to this work

${ }^{+\dagger}$ Bruno Reversade and Heleen $\mathrm{H}$. Arts contributed equally to this work

1 Department of Human Genetics (855), Radboud Institute for Molecular

Life Sciences, Radboud University Medical Centre, PO-Box 9101, 6500

HB Nijmegen, The Netherlands

${ }^{2}$ Laboratory of Human Embryology \& Genetics, Institute of Medical

Biology, A*STAR, Singapore, Singapore

Full list of author information is available at the end of the article
} 


\section{Background}

We have previously described an extended consanguineous family from an Old Order Amish community affected with neonatal lethal endocrine-cerebro-osteodysplasia (ECO) syndrome, characterized primarily by endocrine, cerebral, and skeletal abnormalities [1]. This is the only family with ECO syndrome known to date. Through homozygosity mapping and candidate gene sequencing, we identified a homozygous missense mutation (c.815G > A [UCSC HG19: NM_014920] and p.R272Q [UCSC: NP_055735]) in ICK, which encodes the intestinal cell kinase [1]. The ICK protein belongs to the roscross hybridizing kinase (RCK) family, which consists of two other proteins, i.e., male germ cell-associated kinase (MAK) and MAPK/MAK/MRK overlapping kinase (MOK) [2-4]. Recent studies suggest that ICK is a ciliary protein, involved in ciliogenesis and regulation of intraflagellar transport (IFT) [5-8]. Here, we describe a new family with one affected fetus who was initially diagnosed with short-rib thoracic dysplasia (SRTD), a clinically and genetically heterogeneous group of disorders that are characterized by skeletal abnormalities, including a narrow thorax, short ribs, short and bowed tubular bones, and a number of extraskeletal features [MIM:PS208500]. Homozygosity mapping and whole-exome sequencing in the affected fetus revealed a novel homozygous missense mutation in ICK (c.358G > T; p.G120C), confirming that disruptions in this gene cause ECO syndrome, a disorder that shows marked clinical overlap with the short-rib thoracic dysplasia syndromes (SRTD), Majewski and MohrMajewski in particular [1]. Based on the clinical overlap between ECO syndrome and these other ciliopathies, and recent in vitro and mouse studies $[5,6]$ that showed that ICK is a ciliary protein, we tested whether either mutation (p.G120C or p.R272Q) affects cilium presence, morphology, and function in ciliated mouse Inner Medullary Collecting Duct 3 (mIMCD3) cells and in skin fibroblasts derived from a p.R272Q patient with ECO syndrome.

\section{Methods \\ Collection of human blood samples and ethics consent and permissions}

Blood samples were collected from twenty-two family members (I:1-I:4, II:1-II:15 and III:3-III:5) from family 1 . Genomic DNAs (gDNA) were extracted from blood using a Qiagen kit (Cat\# 74106) and from a fetal skin sample (III:6) by standard procedure. Parents gave their informed consent for study participation, and the study was approved by the Clinical Research Ethics Committees of Singapore (IRB \#2013/1029/E) and Istanbul Medical Faculty with protocol number 2012.743-IRB2.1061.
Collection of human fibroblasts lines and ethics statement A fibroblast cell line from an Old Order Amish patient (family 2 in this paper) with ECO syndrome and two healthy controls from non-Amish (control I) and Amish (control II) communities were collected previously [1]. Tissues were obtained with informed consent, whereby it should be noted that parents provided informed consent for the patient with ECO syndrome. Material was collected with approval by the Office of Research Ethics of the University of Western Ontario with the following reference number: $07920 \mathrm{E}$.

\section{Genotyping and homozygosity mapping}

14 individuals, including I:2, I:4, II:1, II:4, II:8, II:9, II:10, II:11, II:12, II:13, III:3, III:4, III:5, and III:6, were genotyped using Illumina HumanCoreExome-12v1 BeadChips following manufacturer's instructions. Call rates were above $99 \%$, and gender and relationship were verified using Illumina GenomeStudio software. IdenticalBy-Descent (IBD) mapping was performed by searching for homozygous regions in the unique affected individual using custom programs written in Mathematica (Wolfram Research, Inc.). Allowing $1 \%$ error rate, all homozygous regions that were $>2 \mathrm{cM}$ were examined. Candidate regions were further refined by exclusion of common homozygous segments with any of the 13 unaffected family members.

\section{Whole-exome sequencing}

One microgram of high-molecular weight gDNA extracted from fetus III:6 was used for exome capture with ION TargetSeq Exome Kit. DNA was sheared using Covaris M220 Focused-ultrasonicator (Covaris Inc., Woburn, MA, USA) to target an average fragment size of $200 \mathrm{bp}$. Shearing was followed by end repair, ligation of adapters, nick repair, purification, size selection and final amplification prior to exome capture as per TargetSeq protocol. The amplified DNA was cleaned with Ampure $\mathrm{XP}$ reagent (Agencourt, Boston, USA), and the DNA was eluted in $30 \mu \mathrm{l}$ low TE buffer. The libraries were quantified using a Qubit 2.0 Fluorometer (Life Technologies, Carlsbad, CA, USA). The exome library was used for emulsion PCR on an Ion OneTouch System or Ion Chef System (Life Technologies, Carlsbad, CA, USA) following the manufacturer's protocol. Each library was sequenced on an Ion Proton instrument (Life Technologies, Carlsbad, CA, USA) using one ION PI chip.

Sequence reads were aligned to the human reference genome (Human GRCh37 (HG19) build) using Torrent Mapping Alignment Program (TMAP) from the Torrent Suite (v4.2.1). PCR duplicates in the BAM file were identified by the Filter Duplicates plugin (v4.2) and removed. 
The variants were called using the Torrent Variant Caller (TVC) plugin (v4.2.1) and imported into Ion Reporter (v4.2), where each variant was annotated using the "annotate single sample variants" workflow. Information including the associated gene; variant location; quality score; coverage; amino acid change; predicted functional consequences using SIFT [9], PolyPhen2 [10], and Grantham [11]; phyloP conservation scores [12]; and 5000 genomes Minor Allele Frequencies were provided for each variant. Common SNPs referenced in the NCBI database (ftp:// ftp.ncbi.nlm.nih.gov/pub/clinvar/vcf_GRCh37/) and the Exome Sequencing Project (http://evs.gs.washington.edu/ EVS/) were filtered out. Variants that were predicted to be synonymous or not having a location on either a coding exon, UTR, splice site junction, or flanking intron were also removed. Variants were next compared to an in-house database of 138 previously sequenced samples. Those variants that were present in greater than $3 \%$ of the previously sequenced samples were removed.

\section{Cloning}

The ultimate ORF clone of human ICK cDNA (IOH38087) in pENTR221 [1] was used to transfer ICK into p733 (Life Technologies) through Gateway cloning (Life Technologies) according to the manufacturer's instructions. The p733 vector encodes a monomeric Red Fluorescent Protein (mRFP). The p.G120C and p.R272Q mutations were introduced through site-directed mutagenesis, and constructs were validated with restriction digests and Sanger sequencing.

\section{Cell culture}

Localization studies of mutant and wild-type mRFP-ICK were performed in two different cell lines, i.e., Human Embryonic Kidney Cells 293 with Tantigen of SV40 (HEK293T) and mouse inner medullary collecting duct 3 (mIMCD3) cells. HEK293T cells were cultured in Dulbecco's modified eagle's medium (DMEM) with $10 \%$ fetal calf serum (FCS) and mIMCD3 cells in DMEM/F12 (1:1) supplemented with $10 \%$ FCS. Fibroblasts from an Old Order Amish patient with ECO syndrome and two healthy controls from non-Amish (control I) and Amish (control II) were cultured for immunocytochemistry experimentation. Fibroblasts were cultured in DMEM supplemented with $20 \%$ FCS. All cell lines were cultured at standard cell culture conditions.

\section{Transfections in HEK293T and mIMCD3 cells}

To analyze the cellular localization of mRFP-ICK in HEK293T cells, these cells were cultured on sterile coverslips for $24 \mathrm{~h}$. Cells were then transfected with p733 vector DNA encoding either wild-type or mutant (p.R272Q or p.G120C) mRFP-ICK using Lipofectamine (Life Technologies, Bleiswijk, The Netherlands) according to manufacturer's instructions. $48 \mathrm{~h}$ post transfection, cells were fixed in ice-cold methanol for $10 \mathrm{~min}$. After a quick wash with PBS, cells were permeabilized with $0.5 \%$ Triton $\mathrm{X}-100$ in PBS. A drop of DAPI containing FluoromountG (Southern Biotech, Birmingham, AL, USA) was added to the cells, and cover glasses were then placed on microscopic glass slides. The experiment was performed twice, and $>35$ cells were counted per condition. Microscopic analysis was conducted with a Zeiss Axio Imager Z2 fluorescence microscope (Zeiss, Sliedrecht, The Netherlands) equipped with an ApoTome slider. Images were obtained with ZEN 2012 software (Zeiss) and processed with Photoshop CS4 (Adobe Systems, San Jose, CA, USA) and FIJI software. The protocol to determine the ciliary localization of mRFP-ICK in mIMCD3 cells was largely similar compared to HEK293T studies; however, after culturing mIMCD3 cells for $24 \mathrm{~h}$ on sterile coverslips followed by $24 \mathrm{~h}$ of transfection, culture medium was replaced by serum-low medium containing $0.2 \%$ FCS. mIMCD3 cells were grown in this medium for $24 \mathrm{~h}$ to induce ciliogenesis. Subsequently, immunocytochemistry and microscopy were performed. The experiment was performed in quadruplicate, and $>50$ cilia were analyzed per condition.

\section{Immunocytochemistry}

The mutational effects of p.R272Q were analyzed in patient-derived fibroblasts from an affected individual from family 2 compared to fibroblasts from healthy nonAmish (control I) and Amish (control II) controls. The fibroblast cell lines were cultured on sterile coverslips for 24 hours. Subsequently, ciliogenesis was induced by replacing the culture medium for DMEM with $0.2 \%$ FCS for $48 \mathrm{~h}$, followed by immunostaining. For this purpose, cells were washed in PBS, fixed in $2 \%$ paraformaldehyde in PBS for 20 min permeabilized in $1 \%$ triton $\mathrm{X}-100$ in PBS for $5 \mathrm{~min}$. Prior to antibody binding, the cells were blocked for $30 \mathrm{~min}$ in $2 \%$ bovine serum albumin (BSA) in PBS. The following primary antibodies were used: ARL13B (rabbit polyclonal, Proteintech Group, Manchester, United Kingdom, 1:500) and RPGRIP1L (custom-made guinea pig polyclonal; SNC039, 1:500). Primary antibodies were diluted in $2 \%$ BSA/PBS and incubated for $1 \mathrm{~h}$ at room temperature. After washing with PBS, cells were incubated with fluorescentlylabeled secondary antibodies for $30 \mathrm{~min}$. These include anti-guinea pig IgG Alexa Fluor 568 and 647 (1:400), and anti-rabbit IgG Alexa Fluor 488 (1:400). These antibodies were all obtained from Life Technologies (Bleiswijk, The Netherlands). After washing cover glasses in PBS, a drop of DAPI containing Fluoromount-G (Southern 
Biotech, Birmingham, AL, USA) was added to the cells. Cover glasses were placed on microscopic glass slides, and microscopic analysis was performed as previously described.

\section{Results}

\section{Clinical description of patient I}

The affected fetus from family 1 (III:6, patient I) was born to a consanguineous Turkish couple with three unaffected children (III:3, III:4, and III:5), a newborn who died $12 \mathrm{~h}$ after birth (III:1) due to an unknown etiology, and a still birth (SB) baby (III:2) (Fig. 1a). Antenatal ultrasound at 16 weeks of gestation showed cystic hygroma, scalp edema ascites, bilateral ventriculomegaly, very short tubular bones and polydactyly of hands and feet, and short ribs. A differential diagnosis of SRTD was made. Follow-up scan at 19 weeks of gestation revealed large and hyperechogenic kidneys, and a ventricular septal defect. Fetal karyotype was 46 , XY. The pregnancy was terminated at 33 weeks of gestation. In addition to hydropic appearance, short thorax, and very short limbs, postmortem examination revealed oro-facial-digital defects with syndromic craniofacial features such as high and broad forehead, deep set eyes, hypertelorism, very small nose, anteverted nares, midline pseudocleft of the upper lip, clefting of the soft palate, natal teeth, labiogingival fusion, irregular alveolar crest, tongue fused to the lower palate, multiple frenula, micrognathia, low-set and posteriorly rotated ears, postaxial heptadactyly of the hands, and preaxial heptadactyly of the feet. Sex reversal with hypoplastic labial folds and anterior ectopic anus were also noted. Radiographs showed resemblance to SRTD type II, with very short tubular bones, torpedo-like femora, ovoid hypoplastic tibiae, premature epiphyseal ossifications in the lower limbs, short ribs, mildly defective ossification of the vertebrae, and small ilia with normal shape. Figure $1 \mathrm{~b}$ shows various phenotypic characteristics of patient I. The clinical features observed in patient 1 significantly overlap with the features described of the affected infants in family 2 , described by Lahiry et al. [1]. Table 1 shows a phenotypic comparison of the patients from both families.

\section{Genotyping and homozygosity mapping}

Homozygosity mapping identified IBD regions in the unique affected individual that were refined by exclusion of common homozygous segments with any of the 13 unaffected members of family 1 . Four homozygous loci $(>2 \mathrm{CM})$, totaling $50 \mathrm{Mb}$, were delineated on chromosomes chr6: 33, 784, 864-55, 502, 257; chr8: 119, 886, 091-129, 606, 949; chr11: 120, 548, 318-131, 461, 292; and chr20: 29, 834,107-37, 252, 342 (HG19) (Fig. 1c).

\section{Whole-exome sequencing}

Whole-exome sequencing of the fetus III:6 gDNA generated $15.6 \mathrm{~Gb}$ of data with an average read length of $169 \mathrm{bp}$. An average coverage of $194 \mathrm{X}$ was achieved over the exome, with $96 \%$ of bases covered at least 20X. A total of 34,881 variants were identified across proteincoding exons, UTRs, splice sites, and flanking introns. After applying all filters, a final set of 548 variants (49 homozygous and 499 heterozygous) were identified (Additional file 1: Table S1). Only 5 homozygous variants were found in the IBD blocks, of which 4 have been seen in population or affect a non-conserved amino acid, leaving only one new protein-changing variant $(\mathrm{c} .358 \mathrm{G}>\mathrm{T})$ in ICK (NM_014920.3) (Fig. 1c). Genotypes of the 376 flanking SNPs (Chr6: 50, 746, 213-54, 121, 367-HG19) were found homozygous only in the fetus compared to the 13 unaffected relatives (Additional file 2: Table S2). We also confirmed by Sanger sequencing that this ICK mutation was homozygous in the fetus, heterozygous in both parents and either heterozygous, or wild type in 20 other relatives (Additional file 3: Figure S1). To date (October 2015), this variant has not been reported in any public databases such as the online Exome Aggregation Consortium (ExAC) (http://exac.broadinstitute. org). Neither has the variant been reported in 1020 individuals who were whole exome sequenced by the Turkish National Genomics Research groups under TUBITAK. In addition, the p.G120C variant was not found in our inhouse database containing exomes of 72 other Turkish individuals, which further underlines the rare character of this mutation. This missense mutation (p.G120C) leads to the alteration of a conserved glycine (Additional file 4: Figure S2) and is distinct from the ICK mutation p.R272Q previously reported in fetuses and newborns with ECO syndrome [1] (Fig. 1d). Both mutations are located in the serine-threonine protein kinase catalytic domain of ICK, while p.R272Q also affects a nuclear localization signal (Fig. 1d). To conclude, through identification of a second ICK mutation in a fetus who displays ciliopathy related features, we confirm that genetic changes in ICK gene cause ECO syndrome.

\section{Ciliary localization of ICK is altered in ICK mutants}

We studied the cellular and ciliary localization of the two homozygous missense mutations in ICK, c.815G > A, p.R272Q known to cause ECO syndrome, and c.358G > T, p.G120C from the family reported here. The cellular localization of ICK was analyzed using HEK293T cells that were transiently transfected with mRFP-ICK constructs. As previously shown by Lahiry et al., wildtype ICK localized to the nucleus of these cells, whereas the ICK p.R272Q mutant remained in the cytoplasm. On the contrary, the novel ICK mutant p.G120C behaved as 


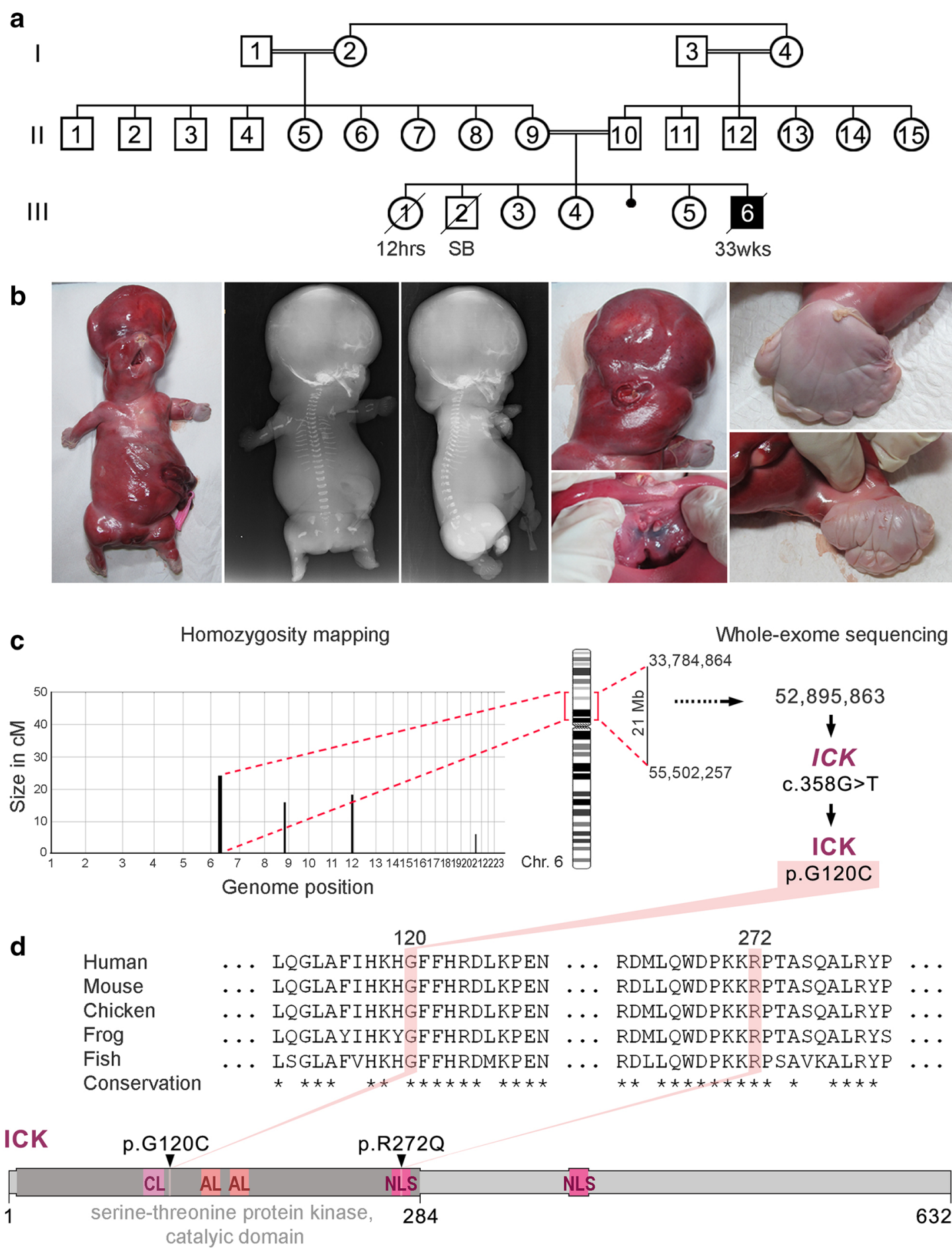

Fig. 1 Phenotypic characteristics of a proband with endocrine-cerebro-osteodysplasia (ECO) syndrome caused by a new missense mutation in ICK. a Pedigree of Family 1. b Photographs and radiographs of the affected fetus. Note hydrops, tetramicromelia, narrow thorax, polydactyly with hypoplastic fingers and toes, and craniofacial anomalies including high forehead, deep set eyes, cleft lip and palate, natal teeth, multiple frenula of the upper lip, and low-set ears. c Homozygosity mapping delineated four candidate regions including one of $21 \mathrm{Mb}$ on chromosome 6 . Wholeexome sequencing of the fetus revealed a homozygous mismatch at position UCSC HG19 chr6: 52, 895, 863, causing one residue change p.G120C in ICK protein (NP_055735). d The mutated residue p.G120C is localized in the catalytic domain of the serine-threonine kinase ICK. The previously indentified variant p.R272Q that was found in ECO fetuses of an Old Order Amish family also resides in this domain. The glycine residue at p.120 is conserved in all vertebrate ICK homologs. CL catalytic loop; AL activation loop; NLS nuclear localization signal 
Table 1 Clinical description and comparison of affected individuals carrying a homozygous mutation in ICK

\begin{tabular}{|c|c|c|}
\hline \multirow[t]{2}{*}{ Clinical features } & \multirow{2}{*}{$\begin{array}{l}\text { Present fetus } \\
\text { Pt I }\end{array}$} & \multirow{2}{*}{$\begin{array}{l}\text { Affected individuals combined/updated } \\
\text { from Lahiry et al. [1] } \\
\text { IV-1, } 2,3,4,7,8 \text {, new }\end{array}$} \\
\hline & & \\
\hline Age at delivery (weeks) & 33 & Range 24-34 \\
\hline Karyotype & $46, X Y$ & $46(6 / 6), X Y(4 / 6), X X(2 / 6)$ \\
\hline Height (centile) & $41 \mathrm{~cm}$ (10-25th) & 3rd-10th (3/5), 90th-97th (2/5) \\
\hline Weight (centile) & $2450 \mathrm{~g}$ (90-97th) & 90th-98th (5/7), 10th-25th (2/7) \\
\hline Head circumference (centile) & $34 \mathrm{~cm}$ (>97th) & $90->98$ th $4 / 6), 3$ rd-20th (2/6) \\
\hline Autopsy & - & $+(3 / 6)$ \\
\hline \multicolumn{3}{|l|}{ Oral } \\
\hline Cleft palate & Cleft soft palate & Midline (5/6), notch in alveolar (1/6) \\
\hline Cleft lip & Midline notching & Midline (5/7), bilateral (2/7) \\
\hline Presence of premaxilla & + & $+(2 / 6)$ \\
\hline Prominent upper lip region & - & $+(2 / 6)$ \\
\hline Hypoplastic/absent epiglottis & Unknown & $+(2 / 3)$ \\
\hline Hypoplastic/absent larynx & Unknown & $+(2 / 3)$ \\
\hline \multicolumn{3}{|l|}{ Craniofacial } \\
\hline Dolichocephaly & - & $+(3 / 6)$ \\
\hline Midface hypoplasia & + & $+(6 / 6)$ \\
\hline Microphthalmia & + & + (4/6), cystic $(2 / 6)$ \\
\hline Retinal dysplasia & Unknown & $+(1 / 3)$ \\
\hline Deep set eyes & + & $+(5 / 5)$ \\
\hline Fused eyelids & - & $+(1 / 6)$ \\
\hline Hypotelorism & - & $+(1 / 1)$ \\
\hline Flat and wide nasal bridge & + & $+(6 / 6)$ \\
\hline Dysplastic and low-set ears & + & $+(5 / 5)$ \\
\hline Micrognathia & + & $+(6 / 6)$ \\
\hline Excess skin below chin & - & $+(4 / 6)$ \\
\hline Single nostril & - & $+(1 / 1)$ \\
\hline Natal teeth & + & $+(1 / 6)$ \\
\hline \multicolumn{3}{|l|}{ Limbs-thorax } \\
\hline Micromelia & + , severe & $+(7 / 7)$ \\
\hline Polydactyly (postaxial) & Upper limbs, heptadactyly & $+(6 / 6)$ \\
\hline Polydactyly (preaxial) & Lower limbs, heptadactyly & - \\
\hline Brachydactyly & +, Severe & $+(6 / 6)$ \\
\hline Syndactyly & 4 limbs, total syndactyly & $+(7 / 7)$ \\
\hline Hitch-hikers'thumbs & - & $+(1 / 6)$ \\
\hline Palmar creases abnormalities & Irregular, vertical creases & $+(3 / 3)$ \\
\hline Ulnar deviation of hands & - & $+(6 / 6)$ \\
\hline Bowing of forearms & + & $+(6 / 6)$ \\
\hline Bowing of lower legs & - & $+(2 / 6)$ \\
\hline Abducted hips & + & $+(6 / 6)$ \\
\hline Sandal gap & - & $+(6 / 6)$ \\
\hline Talipes equinovarus & + & - \\
\hline Chest width & Narrow & Broad (1/6), narrow (1/6) \\
\hline Prominent xyphoid & - & $+(1 / 6)$ \\
\hline \multicolumn{3}{|l|}{ Radiography } \\
\hline Abnormal long bones (radius, ulna, tibia, fibula) & $\begin{array}{l}\text { Very short with bowed radii and more severe } \\
\text { involvement of the lower limb }\end{array}$ & Short diaphysis (1/1) \\
\hline Short and incurved ulnae & + & $+(1 / 1)$ \\
\hline Short and ovoid tibiae & Very short, with very short fibula & $+(1 / 1)$ \\
\hline
\end{tabular}


Table 1 continued

\begin{tabular}{|c|c|c|}
\hline \multirow[t]{2}{*}{ Clinical features } & \multirow{2}{*}{$\begin{array}{l}\text { Present fetus } \\
\text { Pt I }\end{array}$} & \multirow{2}{*}{$\begin{array}{l}\text { Affected individuals combined/updated } \\
\text { from Lahiry et al. [1] } \\
\text { IV-1, } 2,3,4,7,8 \text {, new }\end{array}$} \\
\hline & & \\
\hline Abnormal humerus & Very short & Short diaphysis (1/1) \\
\hline Abnormal femur & Very short, torpedo-like & Short and ovoid (1/1) \\
\hline Spondylar involvement &,+ Mildly defective ossification & Unknown \\
\hline Premature epiphyseal ossification &,+ Knees & Unknown \\
\hline Abnormal ilium & Small ilia with normal contour & $+(1 / 1)$ \\
\hline Abnormal/hypoplastic acetabular roof & - & $+(1 / 1)$ \\
\hline \multicolumn{3}{|l|}{ Central nervous system } \\
\hline Evidence of holoprosencephaly & - & $+(4 / 4)$ \\
\hline Corpus callosum & + & $+(3 / 3)$ \\
\hline Absence of septum pellucidum & + & $+(2 / 2)$ \\
\hline Hydrocephalus (ventriculomegaly) & + & $+(2 / 2)$ \\
\hline Dysmorphic cerebral aqueduct & Unknown & $+(2 / 2)$ \\
\hline Olfactory bulbs & Unknown & $+(2 / 2)$ \\
\hline Cerebral cortex malformation & Unknown & $+(3 / 3)$ \\
\hline Brainstem malformation & Unknown & $+(3 / 3)$ \\
\hline Cerebellar abnormalities & - & $+(2 / 2)$ \\
\hline Hippocampus agenesis & Unknown & $+(2 / 2)$ \\
\hline Leptomeningeal glioneuronal heterotopia & Unknown & $+(1 / 1)$ \\
\hline Spinal cord malformation & Unknown & $+(1 / 2)$ \\
\hline \multicolumn{3}{|l|}{ Endocrine system } \\
\hline Pituitary gland & Unknown & + Absent $(2 / 2)$ \\
\hline Adrenal glands & Unknown & + Absent (1/3), hypoplastic (2/3) \\
\hline \multicolumn{3}{|l|}{ Other } \\
\hline Polyhydramnios & - & $+(1 / 6)$ \\
\hline Fetal hydrops & + & - \\
\hline Pulmonary hypoplasia & Unknown & $+(1 / 1)$ \\
\hline Congenital heart defect &,+ VSD & - \\
\hline Gastrointestinal anomalies & Unknown & $+(2 / 3)$ \\
\hline External genitalia abnormalities & Sex reversal with hypoplastic labial folds & $+(4 / 7)$ \\
\hline Cryptorchidism & $\mathrm{n} / \mathrm{a}$ & $+(1 / 6)^{c}$ \\
\hline (Hyper)echogenic kidneys & + & $+(3 / 6)$ \\
\hline Large kidneys & + & $+(1 / 6)^{d}$ \\
\hline Squamous metaplasia of bladder & Unknown & $+(1 / 3)$ \\
\hline
\end{tabular}

Blank cells indicate that information was unavailable

a Information gathered from attending-physician reports

b Report from International Skeletal Dysplasia Registry (Cedars-Sinai Medical Center) for patient 1

c Feature is reported as patient had ambiguous external genitalia

d Large kidneys displayed cystic tubules

wild-type ICK protein and localized in the HEK293T cell nucleus (Fig. 2a, b). Since the mutation p.R272Q resides in the nuclear localization signal domain of ICK, it may affect the nuclear import of ICK protein. To examine the effect of p.R272Q and p.G120C mutations on ciliogenesis, we transiently transfected mIMCD3 cells with mRFPICK constructs and stimulated the cells to form cilia by serum starvation. While wild-type mRFP-ICK was often present in the ciliary axoneme with or without enrichment at the ciliary base (92\%), both ICK mutants were enriched at the ciliary tip in $\sim 75 \%$ of the cells (Fig. 2c, d). Figure S3 in Additional file 5 visualizes each of the ciliary phenotypes shown in Fig. 2d. Cilia of cells expressing mutant mRFP-ICK display an aberrant bulged tip in $\sim 70 \%$ of ciliated cells as visualized with ARL13B (Fig. 2c), while ciliary length is not essentially altered (Additional 
a

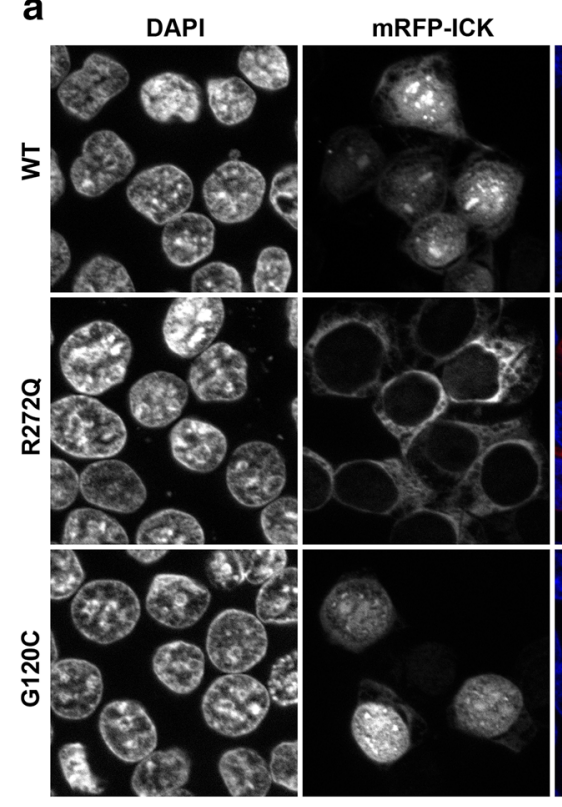

C mRFP-ICK

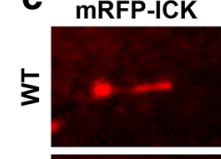

ARL13B
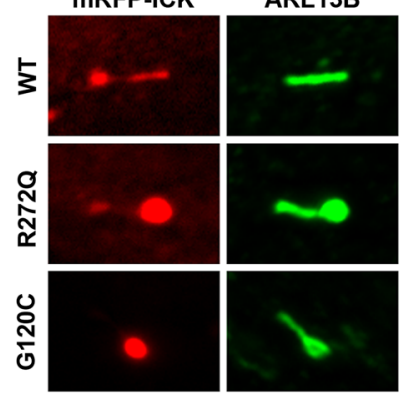

e

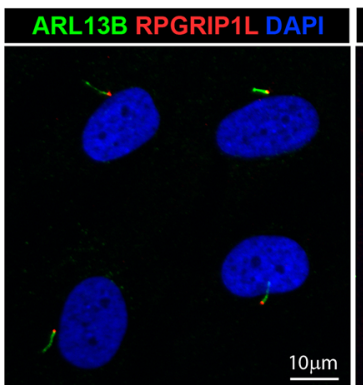

Control I

$\underline{10 \mu \mathrm{m}}$

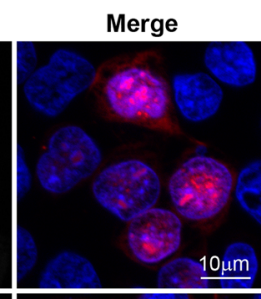

RPGRIP1L
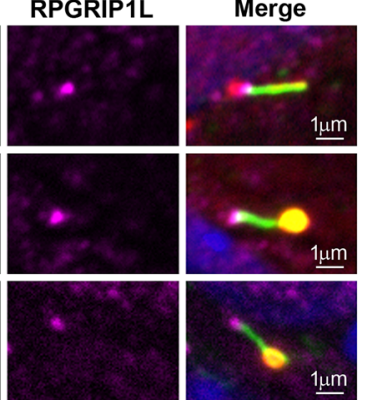

$1 \mu \mathrm{m}$

b

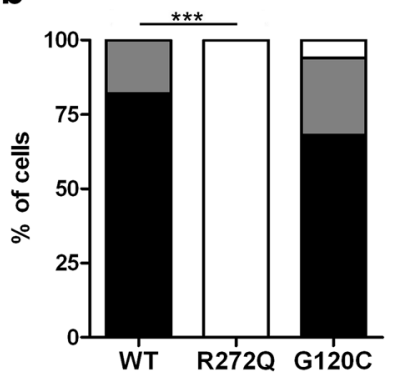

WT R27

$\square$ Nuclear + cytoplasmic

$\square$ Cytoplasmic

$\underline{10 \mu \mathrm{m}}$

$10 \mu \mathrm{m}$

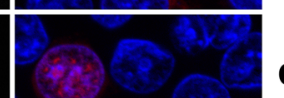

d

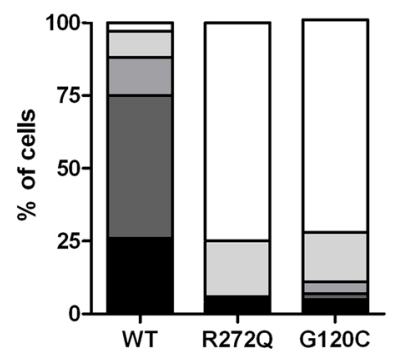

\section{f}

Cilium

$\square$ Cilium + base enrichment

$\square$ Base + tip

$\square$ Tip

$\square$ Tip enrichment

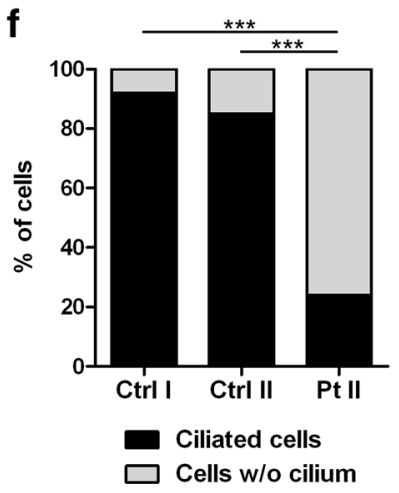


(See figure on previous page.)

Fig. 2 Mutations found in ICK affect ciliogenesis and ciliary localization. a, b The cellular localization of ICK was analyzed in HEK293T cells overexpressing wild-type or two mutated forms of mRFP-ICK: p.R272Q (positive control, mutation previously studied in ECO infants) and p.G120C (new mutation detected in the current study). Nuclei were stained with DAPI (blue) and mRFP-ICK proteins are shown in red. mRFP-ICK with the p.G120C mutation showed the same nuclear localization as wild-type mRFP-ICK transfected cells, while nuclear import was completely lost when mRFP-ICK with the p.R272Q mutation was expressed (Fisher's exact test two-tailed $p<0.0001$, $>35$ cells counted per condition). c, d The ciliary localization of ICK was studied in mIMCD3 cells transiently transfected with wild-type or two mutant forms of mRFP-ICK: p.R272Q or p.G120C. Wild-type mRFPICK mostly localized to the ciliary axoneme and was often enriched at the ciliary base, while both mRFP-ICK mutants enriched at ciliary tips. Ciliary axonemes were visualized with anti-ARL13B (green), ciliary transition zones that are present at the ciliary base were marked with anti-RPGRIP1L (pink), mRFP-ICK constructs were shown in red, and nuclei were stained with DAPI (b/ue). Per construct $>50$ transfected, ciliated cells were counted. e, $\mathbf{f}$ Cilium presence was studied in serum-starved fibroblasts derived from an ECO patient with a homozygous missense mutation in ICK (c.815G > A; p.R272Q) and compared to fibroblasts derived from two healthy unrelated controls. Control I represents a non-Amish individual, while control II is from the Amish community. Ciliogenesis was significantly reduced in fibroblasts from the ECO patient compared to the controls (Fisher's exact test two-tailed $p<0.0001$ for both). At least 80 cells were counted per condition. Ciliary axonemes were visualized with anti-ARL13B (green), ciliary transition zones were marked with anti-RPGRIP1L (red), and nuclei were stained with DAPI (blue)

file 6: Figure S4). In summary, while the nuclear localization of ICK is only compromised by the p.R272Q mutation, we found that the ciliary localization is affected by both p.R272Q and p.G120C mutants.

\section{Ciliogenesis is reduced in ECO patient-derived fibroblasts}

To study the endogenous effect of the homozygous missense mutation p.R272Q on cilia, we monitored ciliogenesis and ciliary length in primary fibroblasts derived from an ECO patient (Patient II) and compared them to cells derived from two healthy controls, one non-Amish (Control I) and the other from the Amish community (Control II). While 92 and $85 \%$ of the control cells were ciliated, only $24 \%$ of patient-derived cells formed cilia (Fig. 2e, f). This was determined with a Fisher's exact two-tailed test that showed $p<0.0001$ when comparing the patient sample to each of the controls. We did not observe any major differences in ciliary length (Additional file 7: Figure S5). In summary, we found that ECO patient-derived fibroblasts display reduced ciliogenesis, while the cilia that form are of normal length.

\section{Discussion}

In this study, we identified a novel homozygous missense mutation (c.358G > T [UCSC HG19: NM_014920] and p.G120C [UCSC: NP_055735]) in the ICK gene in a consanguineous Turkish family with one affected fetus who was initially clinically diagnosed as SRTD type II. After mutation identification, the clinical features of the proband were compared to those of the affected progeny of a previously published Amish family with ECO syndrome who had a different homozygous missense mutation in ICK (p.R272Q) [1]. Although it remains unclear if the Turkish proband had typical endocrine abnormalities seen in ECO syndrome, there was significant clinical overlap with Amish fetuses, which leads us to conclude that we identified the second family with ECO syndrome world-wide. This study thus confirms that aberrations of
ICK can indeed cause severe phenotypes that resemble the short-rib thoracic dysplasia spectrum. Our in vitro studies performed in mIMCD3 cells show that mutant mRFP-ICK with either p.R272Q or p.G120C accumulates in the ciliary tip, while the wild-type protein localizes along the ciliary axoneme with enrichment in the ciliary base. Our findings are in line with the data published by Moon et al. [6] who reported similar localization patterns for both wild-type and mutant ICK (p.R272Q). In contrast, Chaya et al. [5] showed that wild-type ICK localizes to the ciliary tip and that this localization is lost in the p.R272Q mutant. These different observations in ICK localization may be due to variation in the expression level of ICK as well as the type and the position of tags in the protein. Typical accumulations of protein at ciliary tips were also observed in fibroblasts derived from patients with ciliopathies such as asphyxiating thoracic dystrophy [MIM:208500] [13] and cranioectodermal dysplasia [MIM:218330] [14]; cilia of these patients often display abnormal ciliary tip accumulation of intraflagellar transport complex B (IFT-B) proteins. The protein accumulations at ciliary tips may be due to defective IFT and disruption in retrograde intraflagellar transport, i.e., transport occurring from the ciliary tip to its base [14]. Thus, the protein accumulations at the ciliary tip that we observe may also be caused by defective IFT and suggest a role for ICK in the regulation of this process. Recent papers by Broekhuis et al. and Chaya et al. $[5,8]$ indeed indicate that ICK coordinates IFT probably through phosphorylation of KIF3A, a motor protein that drives IFT from the ciliary base towards the tip. Considering the presence of p.G120C and p.R272Q mutations in the serine-threonine protein kinase domain, it is tempting to speculate that both mutations affect phosphorylation of KIF3A, thereby dysregulating IFT. While various reports suggest that ICK is a regulator of cilia length $[5,6,8]$, we did not observe dramatic differences in the length of cilia expressing mutant ICK nor in those cilia occurring on 
the apical surface of cells derived from an ECO patient (Additional file 6: Figure S4 and Additional file 7: Figure S5). This may be in part be explained by the likely hypomorph effects of the ECO syndrome-associated missense mutations. We did, however, observe a significantly reduced number of cilia in ECO patient-derived fibroblasts compared to two healthy controls. This may explain the previously described hyperproliferative behavior of these cells [15]. Our reasoning is that cilium formation depends on docking of the mother centriole of the centrosome to the plasma membrane from which the cilium can then emerge. When cilia are unstable or do not form, such as in ECO patient-derived cells, centrioles become available for assembly of the mitotic spindle promoting cell division. The ciliogenesis defect seen in ECO patient-derived fibroblasts is comparable to the defects observed in Ick-deficient mouse embryonic fibroblasts (MEFs), recently described by Chaya et al. [5].

Loss of Ick results in aberrant ciliary Hedgehog signaling in multiple murine organs and fibroblasts $[5,6]$, and this pathway is also dysregulated in other ciliary chondrodysplasias that share clinical features with ECO syndrome and SRTD [16-19]. It is currently unclear how the Hedgehog pathway is affected upon Ick depletion. One hypothesis is that loss of Ick directly affects IFT thereby altering Hedgehog signaling [5]. Another hypothesis is that Ick depletion impacts mTORC1 signaling thereby causing an imbalance in ciliary tubulin synthesis resulting in structural ciliary defects that in turn impact ciliary signaling [8]. In any case, many ECO and SRTD syndrome-associated anomalies are probably due to disturbances of ciliary signaling cascades regulating human development, including but probably not limited to the Hedgehog pathway.

\section{Conclusions}

In conclusion, we here report a novel ICK mutation in a family with ECO syndrome. This is the first replicative allele, which confirms that ICK mutations are indeed associated with severe SRTD resembling phenotypes. We found ciliogenesis defects in fibroblasts derived from a previously reported individual affected by ECO syndrome and noted that expression of mutant ICK (p.R272Q and p.G120C) results in aberrant localization of ICK in ciliary tips. Although ciliary phenotypes vary slightly between cells and species, our findings are in agreement with recent reports on Ick knockout mice and murine Ick depleted cells that reveal a role for Ick in ciliogenesis, ciliary transport, and regulation of cell signaling. Our study is the first to show that ciliary defects occur in cells derived from a patient with ECO syndrome with mutated $I C K$ and thereby provides additional support for inclusion of ECO syndrome in the severe ciliary disease spectrum.

\section{Additional files}

\author{
Additional file 1: Table S1. Filtering of WES data.
}

Additional file 2: Table S2. SPN genotypes flanking the p.G120C mutation present in the fetus and 13 unaffected relatives.

Additional file 3: Figure S1. The ICK missense mutation (c.358G > T; p.G120C) segregates with disease.

Additional file 4: Figure S2. Alignment of the protein kinase domain in ICK, MAK and MOK in different species.

Additional file 5: Figure S3. Different classes of ciliary phenotypes.

Additional file 6: Figure S4. Ciliary length is not essentially altered in cilia of cells expressing mutant mRFP-ICK.

Additional file 7: Figure S5. Ciliary length is not markedly altered in ECO patient-derived fibroblasts.

\section{Abbreviations}

ECO: endocrine-cerebro-osteodysplasia; ExAC: Exome Aggregation Consortium; IBD: identical-by-descent; ICK: intestinal cell kinase; IFT: intraflagellar transport; IFT-B: intraflagellar transport complex B; MAK: male germ cellassociated kinase; MOK: MAPK/MAK/MRK overlapping kinase; MEFs: mouse embryonic fibroblasts; MIM: Mendelian inheritance in man; mTORC1: mammalian target of rapamycin complex 1; RCK: ros-cross hybridizing kinase; SRTD: short-rib thoracic dysplasia; UCSC: UCSC Genome Browser.

\section{Authors' contributions}

$A E, H L$, and SFN performed homozygosity mapping. ST, AYJN, and BV performed whole-exome sequencing, and CB analyzed the data. MMO, DAM, $\mathrm{KMW}$, and NPdW conducted antibody stainings in cells derived from healthy controls and an individual with ECO syndrome. MMO, DAM, and NPdW analyzed the data. MMO conducted transfections and antibody stainings in HEK293T and mIMCD3 cells and analyzed the data. UA and HK diagnosed the SRTD disease and collected blood and skin samples, and informed consent from family 1. CAR, PL, and VMS collected patient cells and informed consent from the family with ECO syndrome. VMS, HHA, and BR designed the study. $M M O, C B, D A M$, and HHA wrote the manuscript, and VMS, GJP, RAH, RR, and BR reviewed the manuscript critically. All the authors read and approved the final manuscript.

\section{Author details}

1 Department of Human Genetics (855), Radboud Institute for Molecular Life Sciences, Radboud University Medical Centre, PO-Box 9101, 6500 HB Nijmegen, The Netherlands. ${ }^{2}$ Laboratory of Human Embryology \& Genetics, Institute of Medical Biology, A*STAR, Singapore, Singapore. ${ }^{3}$ Medical Genetics Department, Istanbul Medical Faculty, Istanbul University, Istanbul, Turkey. ${ }^{4}$ Institute of Molecular and Cell Biology, A*STAR, Singapore, Singapore. ${ }^{5}$ Department of Human Genetics, David Geffen School of Medicine, University of California, Los Angeles, USA. ${ }^{6}$ Department of Pathology and Laboratory Medicine, David Geffen School of Medicine, University of California, Los Angeles, USA. ${ }^{7}$ Department of Biochemistry, University of Western Ontario, Room 4212A, 1151 Richmond Street N, N6A $5 B 7$ London, ON, Canada. ${ }^{8}$ Medical Genetics Program, London Health Sciences Centre, London, ON, Canada. ${ }^{9}$ Children's Health Research Institute, London, ON, Canada. ${ }^{10}$ Department of Paediatrics, The Hospital for Sick Children, Toronto, ON, Canada. ${ }^{11}$ Program in Molecular Medicine, University of Massachusetts Medical School, Worcester, MA, USA. ${ }^{12}$ Robarts Research Institute, London, ON, Canada. ${ }^{13}$ Medical Genetics Department, Koç University School of Medicine, Istanbul, Turkey.

\section{Acknowledgements}

We thank Dr. Hanka Venselaar for useful discussions on the molecular structure of ICK and the families for participation in our study. Written informed consent was obtained from the parents of the patient for publication of their individual details and accompanying images in this manuscript. The consent form is held by the authors and is available for review by the Editor-in-Chief. This study was funded by the Dutch Kidney Foundation (KOUNCIL consortium project 
(P11.18 to HHA) and the Netherlands Organization for Health Research and Development (ZonMW Veni-91613008 to HHA) and the Netherlands Organization for Scientific research (NWO Vici-016130664 to RR). This work was also funded by a Strategic Positioning Fund on Genetic Orphan Diseases from A*STAR, Singapore, and the Scientific and Technological Research Council of Turkey (TUBITAK) by 112 S398 to HK (E-RARE network CRANIRARE-2).

\section{Competing interests}

The authors declare that they have no competing interests.

Received: 3 September 2015 Accepted: 27 January 2016 Published online: 11 April 2016

\section{References}

1. Lahiry P, Wang J, Robinson JF, Turowec JP, Litchfield DW, Lanktree MB, et al. A multiplex human syndrome implicates a key role for intestinal cell kinase in development of central nervous, skeletal, and endocrine systems. Am J Hum Genet. 2009;84(2):134-47. doi:10.1016/j. ajhg.2008.12.017.

2. Abe S, Yagi T, Ishiyama S, Hiroe M, Marumo F, Ikawa Y. Molecular cloning of a novel serine/threonine kinase, MRK, possibly involved in cardiac development. Oncogene. 1995;11(11):2187-95.

3. Bladt F, Birchmeier C. Characterization and expression analysis of the murine rck gene: a protein kinase with a potential function in sensory cells. Differentiation; research in biological diversity. 1993;53(2):115-22.

4. Gopalan G, Centanni JM, Moss S, Donovan PJ. A novel mammalian kinase, T/STK 30, is highly expressed in the germ line. Mol Reprod Dev. 1999;52(1):9-17. doi:10.1002/ (SICI)1098-2795(199901)52:1<9:AID-MRD2>3.0.CO;2-O.

5. Chaya T, Omori Y, Kuwahara R, Furukawa T. ICK is essential for cell typespecific ciliogenesis and the regulation of ciliary transport. EMBO J. 2014;33(11):1227-42. doi:10.1002/embj.201488175.

6. Moon H, Song J, Shin JO, Lee H, Kim HK, Eggenschwiller JT, et al. Intestinal cell kinase, a protein associated with endocrine-cerebro-osteodysplasia syndrome, is a key regulator of cilia length and Hedgehog signaling. Proc Natl Acad Sci USA. 2014;111(23):8541-6. doi:10.1073/pnas.1323161111.

7. Yang Y, Roine N, Makela TP. CCRK depletion inhibits glioblastoma cell proliferation in a cilium-dependent manner. EMBO Rep. 2013;14(8):741-7. doi:10.1038/embor.2013.80.

8. Broekhuis JR, Verhey KJ, Jansen G. Regulation of cilium length and intraflagellar transport by the RCK-kinases ICK and MOK in renal epithelial cells. PLoS One. 2014;9(9):e108470. doi:10.1371/journal.pone.0108470.
9. Kumar P, Henikoff S, Ng PC. Predicting the effects of coding non-synonymous variants on protein function using the SIFT algorithm. Nat Protoc. 2009;4(7):1073-81. doi:10.1038/nprot.2009.86.

10. Adzhubei IA, Schmidt S, Peshkin L, Ramensky VE, Gerasimova A, Bork P, et al. A method and server for predicting damaging missense mutations. Nat Methods. 2010;7(4):248-9. doi:10.1038/nmeth0410-248.

11. Grantham R. Amino acid difference formula to help explain protein evolution. Science. 1974;185(4154):862-4.

12. Pollard KS, Hubisz MJ, Rosenbloom KR, Siepel A. Detection of nonneutral substitution rates on mammalian phylogenies. Genome Res. 2010;20(1):110-21. doi:10.1101/gr.097857.109.

13. Schmidts M, Arts HH, Bongers EM, Yap Z, Oud MM, Antony D, et al. Exome sequencing identifies DYNC2 $\mathrm{H} 1$ mutations as a common cause of asphyxiating thoracic dystrophy (Jeune syndrome) without major polydactyly, renal or retinal involvement. J Med Genet. 2013;50(5):309-23. doi:10.1136/jmedgenet-2012-101284.

14. Arts HH, Bongers EM, Mans DA, van Beersum SE, Oud MM, Bolat E, et al. C14ORF179 encoding IFT43 is mutated in Sensenbrenner syndrome. J Med Genet. 2011;48(6):390-5. doi:10.1136/jmg.2011.088864.

15. Lahiry P, Lee $L$, Frey BJ, Rupar CA, Siu VM, Blencowe BJ, et al. Transcriptional profiling of endocrine cerebro-osteodysplasia using microarray and next-generation sequencing. PLoS ONE. 2011;6(9):e25400. doi:10.1371/ journal.pone.0025400.

16. Ashe A, Butterfield NC, Town L, Courtney AD, Cooper AN, Ferguson C, et al. Mutations in mouse Ift144 model the craniofacial, limb and rib defects in skeletal ciliopathies. Hum Mol Genet. 2012;21 (8):1808-23. doi:10.1093/hmg/ddr613.

17. Cortellino S, Wang C, Wang B, Bassi MR, Caretti E, Champeval D, et al. Defective ciliogenesis, embryonic lethality and severe impairment of the Sonic Hedgehog pathway caused by inactivation of the mouse complex A intraflagellar transport gene Ift122/Wdr10, partially overlapping with the DNA repair gene Med1/Mbd4. Dev Biol. 2009;325(1):225-37. doi:10.1016/j.ydbio.2008.10.020.

18. Huangfu D, Liu A, Rakeman AS, Murcia NS, Niswander L, Anderson KV. Hedgehog signalling in the mouse requires intraflagellar transport proteins. Nature. 2003;426(6962):83-7. doi:10.1038/nature02061.

19. Rix S, Calmont A, Scambler PJ, Beales PL. An Ift80 mouse model of short rib polydactyly syndromes shows defects in hedgehog signalling without loss or malformation of cilia. Hum Mol Genet. 2011;20(7):1306-14. doi:10.1093/hmg/ddr013.

\section{Submit your next manuscript to BioMed Central and we will help you at every step:}

- We accept pre-submission inquiries

- Our selector tool helps you to find the most relevant journal

- We provide round the clock customer support

- Convenient online submission

- Thorough peer review

- Inclusion in PubMed and all major indexing services

- Maximum visibility for your research

Submit your manuscript at www.biomedcentral.com/submit
() Biomed Central 